\title{
Early Venous Thromboembolic Event Prophylaxis in Traumatic Brain Injury with Low-Molecular-Weight Heparin: Risks and Benefits
}

\author{
Roy R. Dudley, ${ }^{4}$ Ishtiaque Aziz, ${ }^{2,4}$ André Bonnici, ${ }^{3}$ Rajeet S. Saluja, ${ }^{4}$ Julie Lamoureux, \\ Boaz Kalmovitch, ${ }^{6}$ Ash Gursahaney, ${ }^{7}$ Tarek Razek, ${ }^{8}$ Mohammad Maleki,,9 and Judith Marcoux ${ }^{1,9}$
}

\begin{abstract}
Traumatic brain injury (TBI) patients are known to be at high risk for venous thromboembolic events (VTEs). The Brain Trauma Foundation Guidelines (2007) state that low-molecular-weight heparin or unfractionated heparin should be used to prevent VTE complications, but suggest that there is an increased risk of expansion of intracranial hemorrhages (ICH) with VTE prophylaxis. In addition, it is unclear which treatment regimen (i.e., medication, dose, and timing) provides the best risk:benefit ratio in TBI patients. We reviewed all moderate-tosevere TBI patients admitted over a 5-year period to: (1) examine the occurrence of VTEs and their timing; (2) examine the symptomatic expansion of ICH while on VTE prophylaxis; and (3) compare the efficacy of two prophylactic agents: enoxaparin and dalteparin. Two-hundred eighty-seven patients were included. VTE prophylaxis was started $48-72 \mathrm{~h}$ post-trauma in all individuals who had no confounding coagulopathy, when two consecutive computed tomography (CT) scans revealed hemorrhage stability. VTEs occurred in $7.3 \%$ of treated patients, mostly within 2 weeks after trauma. Proximal VTEs occurred in $3.1 \%$ of treated patients. No significant difference in VTE rates was seen between enoxaparin $(7.0 \%)$ and dalteparin $(7.5 \% ; p=0.868)$. Moreover, the group treated with dalteparin was more severely injured (higher Injury Severity Score $[p=0.002]$ ), had lower Glasgow Coma Scale (GCS) scores $(p=0.003)$, and had more inferior vena cava (IVC) filters placed $(p=0.007)$. The two groups did not show significant differences in the development of VTE when controlled for ISS and IVC filters $(p=0.819)$. Importantly, only one patient suffered a symptomatic expansion of ICH while on VTE prophylaxis, at 15 days post-trauma. These results suggest that current regimens of VTE prophylaxis used in our TBI population provide a relatively high level of protection against VTEs, and an extremely low risk of expanding ICH. They also suggest that there was no difference in VTE between dalteparin- and enoxaparin-treated patients.
\end{abstract}

Key words: dalteparin; enoxaparin; low-molecular-weight heparin; traumatic brain injury; venous thromboembolic event

\section{Introduction}

$\mathbf{T}$ RAUMATIC BRAIN INJURY (TBI) patients are highly susceptible to the development of venous thromboembolic events (VTEs), including deep veins thromboses (DVTs) and pulmonary emboli (PEs). VTEs occur in up to $25 \%$ of isolated brain injury patients, and up to $50 \%$ of polytrauma patients with brain injuries (Geerts et al., 1996; Rogers et al., 2002).

\footnotetext{
${ }^{1}$ Montreal Neurological Hospital, and ${ }^{9}$ Assistant Professor, Department of Neurosurgery, McGill University Health Centre, Montreal, Quebec, Canada.

${ }^{2}$ Department of Biochemistry, McGill University, Faculty of Sciences, Montreal, Quebec, Canada.

${ }^{3}$ Assistant Chief Pharmacist, McGill University Health Centre, and Associate Clinical Professor, Université de Montréal, Faculty of Pharmacy, Montreal, Quebec, Canada.

${ }^{4}$ Montreal Neurological Hospital, McGill University Health Centre, Montreal, Quebec, Canada.

${ }^{5}$ Statistician, Université de Montréal, Médecine sociale et préventive, Montreal, Quebec, Canada.

${ }^{6}$ Department of Surgery A, E. Wolfson Medical Center, Holon, and Tel Aviv University, Tel Aviv, Israel.

${ }^{7}$ Director, Clinical Services, Adult Critical Care, McGill University Health Center, and Associate Professor, Division of Critical Care, Department of Medicine, McGill University, Montreal, Quebec, Canada.

${ }^{8}$ Director, McGill University Health Center Trauma Program, and Assistant Professor of Surgery, McGill University, McGill University Health Centre, Montreal, Quebec, Canada.
} 
Pulmonary embolism is the third leading cause of death in trauma after the first $24 \mathrm{~h}$ (Smith et al., 1994).

Recently, it has been suggested that TBI itself is an independent risk factor for the development of VTEs. The reasons for this are thought to be threefold: (1) the lengthy period of immobilization suffered by TBI patients due to their frequent comatose or semi-comatose state, as well as other debilitating injuries in the case of polytrauma patients; (2) delay in starting VTE prophylaxis once an intracranial hemorrhage (ICH) has been documented, for fear of the catastrophic consequences of propagating the bleed within the "closed-box" of the intracranial space; and (3) a hypercoagulable state due to tissue factor release (which is believed to exist at the highest levels in the brain), as well as elevated levels of other pro-coagulants such as von Willebrand factor and activated platelets (Brain Trauma Foundation, 2007; Geerts et al., 2004; Gerlach et al., 2003; Reiff et al., 2009).

Based on level 3 evidence, the Brain Trauma Foundation Guidelines for the management of severe traumatic brain injury (2007) suggest that unfractionated heparin (UFH) or lowmolecular-weight heparin (LMWH) be used in combination with mechanical prophylaxis in TBI patients. However, the guidelines warn that there is an increased risk of $\mathrm{ICH}$ expansion with these medications, and recognize that there is insufficient evidence regarding which treatment regimen (medication, dose, and timing) should be used.

Since the randomized controlled trial of LMWH versus UFH as VTE prophylaxis in trauma patients by Geerts and associates (1996) showed a greater risk reduction with LMWH, it has become the preferred prophylactic agent for trauma patients in general. However, this landmark study did not include patients with frank ICH. In fact, of the very small number of patients with bleeding complications in that study, only one patient had an ICH while on the LMWH. Therefore, it provided little guidance for VTE prophylaxis in TBI patients per se.

Few studies have looked at medical VTE prophylaxis in TBI patients, and none have compared different prophylactic agents (Kim and Brophy, 2009; Kleindienst et al., 2003; Norwood et al., 2002, 2008). In addition, the previous studies looked at the risks and benefits of VTE prophylaxis in all patients, regardless of injury severity or Glasgow Coma Scale (GCS) score (Teasdale and Jennet, 1974), or did not comment on injury severity at all. However, less-severely-injured patients are mobilized earlier, and as such are at much lower risk of developing VTEs.

Therefore, we performed a 5-year review of the use of LMWH VTE prophylaxis at our institution. Our specific objectives were: (1) to examine the risks and benefits of LMWH use in our population of moderate-to-severe TBI patients (i.e., the percentage of VTEs that occurred and their timing, and any expansion of ICH seen while on these medications); and (2) to perform a head-to-head comparison of enoxaparin and dalteparin with regard to these risks and benefits in this specific TBI population.

\section{Methods}

\section{Chart review overview}

The Montreal General Hospital (MGH), part of the McGill University Health Centre (MUHC), is one of only three adult tertiary (level 1) trauma centers serving the province of
Quebec, Canada, which has a population of almost 8 million people. The MGH TBI Database was used to identify all patients with a diagnosis of moderate or severe TBI (defined as having a post-resuscitation GCS score between 3 and 12), who were admitted between January 1, 2004 and December 31, 2008. We performed a retrospective study of LMWH VTE prophylaxis in the 694 consecutive cases of moderate-tosevere TBI identified during this 5-year period. The MUHC Ethics Review Board and the Director of Professional Services approved this study, and the informed consent requirement was waived.

\section{Data collected}

The variables of interest that were collected included: (1) demographic data (age and gender); (2) injury-related data (initial GCS score, mechanism of injury, polytrauma versus isolated head injury, and Injury Severity Score [ISS]); (3) any surgical interventions that took place (excluding external ventricular drain insertion); (4) whether an inferior vena cava (IVC) filter was used; (5) if and when a VTE (DVT or PE) was diagnosed, along with the exact anatomical location of the VTE and whether it caused death; (6) if and when an expansion of an ICH occurred and whether this caused death or disability; and (7) the type, dose, dosing schedule, start date, and end date of all medical VTE prophylactic agents used.

\section{Exclusion criteria}

Charts were excluded from data collection and/or analysis for the following reasons: (1) the patient died within $72 \mathrm{~h}$ of admission, and therefore was never treated with VTE prophylaxis for any meaningful period of time; (2) patients who survived but were never treated (many of these patients did well early and were mobilized early, thus avoiding prophylactic anticoagulation; others had persistent contraindications to the prophylactic use of LMWH); (3) patients treated with $\mathrm{UFH}$; (4) patients treated with LMWH, but with an atypical dosing or timing schedule (e.g., enoxaparin $40 \mathrm{mg}$ once a day); (5) patients that were initially $(>24 \mathrm{~h})$ treated abroad, but were eventually transferred to our hospital; and (6) charts that were missing after multiple attempts to locate them.

\section{VTE prophylaxis protocol}

All non-ambulatory patients had mechanical prophylaxis (graduated compression stockings and intermittent pneumatic compression stockings), unless there was a contraindication, such as a lower extremity external fixation device. Patients were also started on LMWH (either enoxaparin $30 \mathrm{mg}$ subcutaneously twice per day, or dalteparin $5000 \mathrm{U}$ subcutaneously once per day) at 48-72 h post-trauma, if they had no confounding coagulopathy, and had two or more consecutive CT scans revealing hemorrhage stability. The timing of 48$72 \mathrm{~h}$ was chosen because there is evidence suggesting that delaying VTE prophylaxis for more than 4 days increases the risk of VTE in major trauma patients threefold (Nathens et al., 2007). At our institution, formal VTE prophylaxis guidelines for trauma patients (based on the randomized controlled trial of Geerts et al., 1996) were established in 2003, which recommended subcutaneous enoxaparin. However, due to lower costs and the convenience of once-a-day injection, as well as an assumed "class-effect" of all LMWH products, 
these institutional guidelines were changed to recommend subcutaneous dalteparin as opposed to enoxaparin. In the absence of evidence to suggest that one medication is better than the other, both medications have been used in parallel in our hospital for more than 5 years. The choice of the LMWH agent was therefore made according to institutional guidelines. The LMWH was continued until the patients were fully ambulatory, except for brief interruptions (usually 6-12 h) for surgery or other invasive intensive care unit (ICU) procedures. IVC filters were also prophylactically placed in selected patients for polytrauma with lower limb long-bone fractures or pelvic fractures.

\section{Outcome measures}

The primary outcome measures were: (1) the percentage of patients who were diagnosed with VTEs; (2) the percentage of patients who were diagnosed with a symptomatic expansion of pre-existing ICH while on LMWH VTE prophylaxis; and (3) any differences between enoxaparin and dalteparin in terms of the percentage of VTEs and expansions of ICH occurring while on these medications. Secondary outcome measures were: (1) differences between enoxaparin and dalteparin in terms of the anatomical location of the VTEs; and (2) timing of VTE diagnosis (i.e., how long after the trauma the VTE was diagnosed). DVTs were diagnosed by duplex ultrasonography of the limbs or neck. Per the ultrasonographic definition used in our hospital, basilic and cephalic vein thrombi in the upper limbs, and greater and lesser saphenous vein thrombi in the lower limbs were not considered DVTs. DVT was defined as a venous thrombosis in the iliac, femoral, popliteal, or posterior tibial veins, or at the trifurcation point of the peroneal, anterior, or posterior tibial veins of the lower extremity; and a venous thrombosis in the internal jugular, innominate, subclavian, axillary, or brachial veins of the upper body. A proximal DVT was defined as a thrombosis in the iliac, femoral, popliteal, internal jugular, innominate, or subclavian veins. PE was diagnosed by spiral chest CT scan. Diagnostic tests were performed after clinical suspicion deemed these tests necessary; no routine screening was performed, in accordance with current guidelines (Geerts et al., 2004). There were no major changes in practice or protocols that might have affected the outcomes during the time of the study period, other than the change in LMWH agent used for VTE prophylaxis in our institution. CT scan of the head was used to diagnose expansion of a pre-existing ICH. Patients had brain imaging when clinically indicated, so not every patient had CT scans while on LMWH. But all brain CT scans while on LMWH were reviewed for any change in previous ICH or new ICH.

\section{Statistical analysis}

Descriptive statistics were reported for all variables as means and standard deviations for numerical variables, and percentages for categorical data. Analyses were done using the statistical computer software program PASW Statistics (SPSS Inc., Chicago, IL). Differences between numbers of

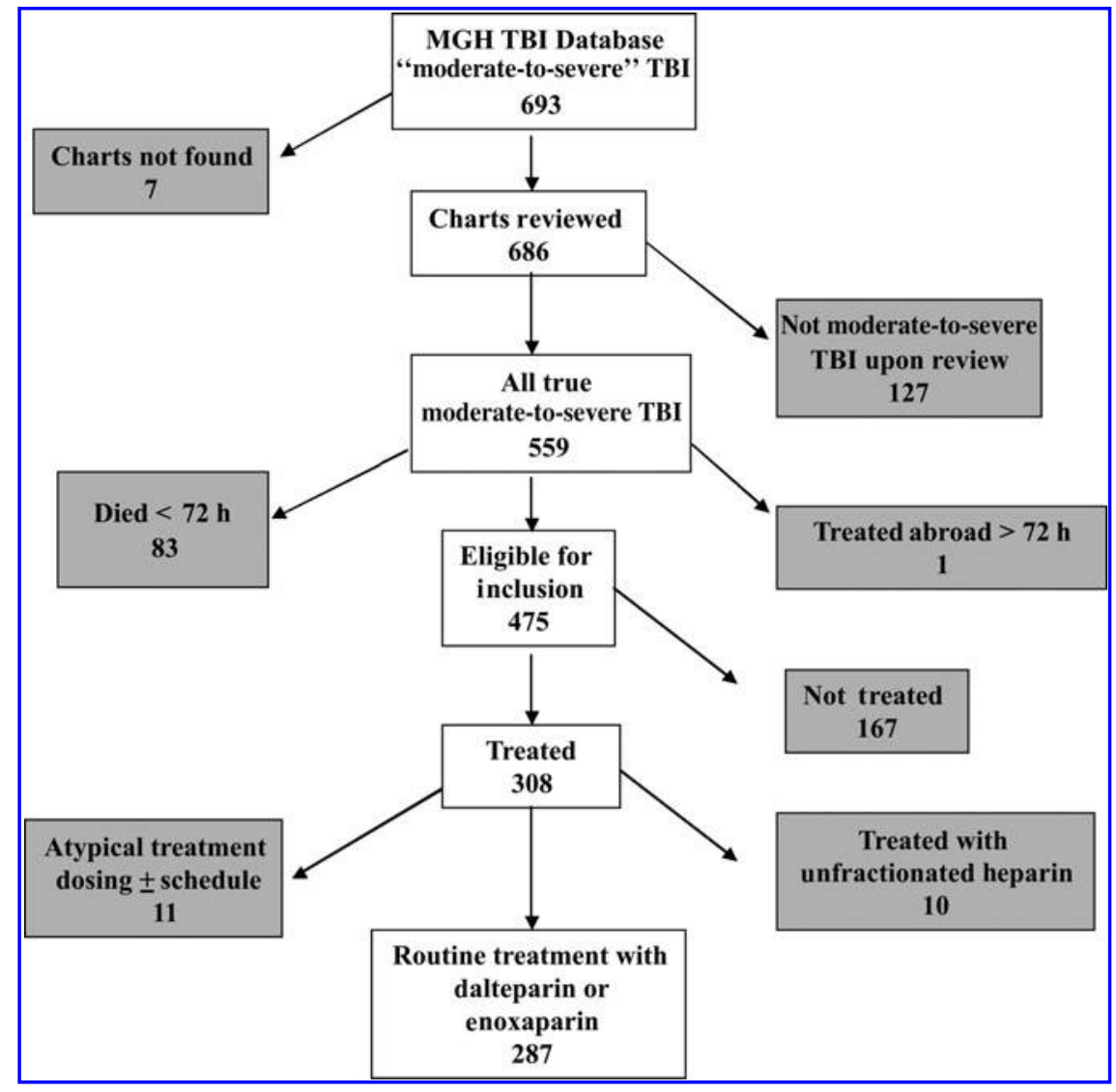

FIG. 1. Diagram of the detailed chart exclusion process (TBI, traumatic brain injury; MGH, Montreal General Hospital). 
Table 1. Patient Characteristics According to the Presence or Absence of Venous Thromboembolic Events

\begin{tabular}{|c|c|c|c|c|}
\hline & Total $\pm S D$ & No $V T E \pm S D$ & $V T E \pm S D$ & Test and $\mathrm{p}$ value \\
\hline Number of treated patients & 287 & 266 & 21 & \\
\hline \multicolumn{5}{|l|}{ Gender } \\
\hline Male & $214(74.6 \%)$ & $195(73.3 \%)$ & $19(90.5 \%)$ & \multirow{5}{*}{$\begin{aligned} \chi_{1 \mathrm{df}}^{2} & =3.025 \\
p & =0.082 \\
\mathrm{t}_{285} & =0.251 \\
p & =0.802 \\
\mathrm{t}_{285} & =1.170 \\
p & =0.243 \\
\mathrm{t}_{285} & =0.172 \\
p & =0.864\end{aligned}$} \\
\hline Female & $73(25.4 \%)$ & $71(26.7 \%)$ & $2(9.5 \%)$ & \\
\hline Average age (years) & $46.5 \pm 20.5$ & $46.5 \pm 20.7$ & $47.6 \pm 19.2$ & \\
\hline Average initial GCS score & $7.4 \pm 3.0$ & $7.3 \pm 3.0$ & $8.1 \pm 3.0$ & \\
\hline Average Injury Severity Score & $33.2 \pm 10.6$ & $33.2 \pm 10.5$ & $33.6 \pm 12.1$ & \\
\hline \multicolumn{5}{|l|}{ Mechanism of injury } \\
\hline Motor vehicle crash & $103(35.9 \%)$ & $96(36.1 \%)$ & $7(33.3 \%)$ & \multirow{5}{*}{$\begin{aligned} \chi_{\mathrm{ldf}}^{2} & =1.924 \\
p & =0.750\end{aligned}$} \\
\hline Fall & $98(34.1 \%)$ & $91(34.2 \%)$ & $7(33.3 \%)$ & \\
\hline Motor vehicle-hit & $51(17.8 \%)$ & $46(17.3 \%)$ & $5(23.8 \%)$ & \\
\hline Assault & $29(10.1 \%)$ & $28(10.5 \%)$ & $1(4.8 \%)$ & \\
\hline Other & $6(2.1 \%)$ & $5(1.9 \%)$ & $1(4.8 \%)$ & \\
\hline Polytrauma & $198(69.0 \%)$ & $185(69.5 \%)$ & $13(61.9 \%)$ & \multirow{4}{*}{$\begin{aligned} \chi_{1 \mathrm{df}}^{2} & =0.532 \\
p & =0.466 \\
\chi^{2}{ }_{1 \mathrm{df}} & =0.255 \\
p & =0.613 \\
\chi_{1 \mathrm{df}} & =0.025 \\
p & =0.874\end{aligned}$} \\
\hline Isolated head injury & $89(31.0 \%)$ & $81(30.5 \%)$ & $8(38.1 \%)$ & \\
\hline Any surgery done & $192(66.9 \%)$ & $179(67.3 \%)$ & $13(61.9 \%)$ & \\
\hline Prophylactic IVC filter & $51(17.8 \%)$ & $47(17.7 \%)$ & $4(19.0 \%)$ & \\
\hline \multicolumn{5}{|l|}{ VTE prophylactic drug } \\
\hline Dalteparin & $159(55.4 \%)$ & $147(55.3 \%)$ & $12(57.1 \%)$ & \multirow{3}{*}{$\begin{aligned} \chi_{1 \mathrm{df}}^{2} & =0.028 \\
p & =0.868\end{aligned}$} \\
\hline Enoxaparin & $128(44.6 \%)$ & $119(44.7 \%)$ & $9(42.9 \%)$ & \\
\hline Intracranial bleed while treated & $1(0.35 \%)$ & $1(0.38 \%)$ & $0(0.0 \%)$ & \\
\hline
\end{tabular}

GCS, Glasgow Coma Scale; IVC, inferior vena cava; SD, standard deviation; VTE, venous thromboembolic event.

patients in the various groups and subgroups (i.e., proportions) were assessed using chi-square analysis, while group means were compared using independent two-tailed $t$-tests. Statistical significance was set at $p<0.05$. In order to control for the differences in baseline characteristics between the enoxaparin and dalteparin groups, we ran a generalized linear model with a binomial probability distribution for the dependent variable (development of a VTE), and a logit link function.

\section{Results}

The MGH TBI database contained 693 charts coded as "moderate-to-severe TBI" (i.e., GCS score 3-12). See Figure 1 for the details of the exclusion process. A total of 287 patient charts were considered eligible for this study. Patient age ranged from 17-92 years. The initial Injury Severity Score (ISS) varied between 4 and 66. According to GCS scores, 187 subjects were considered severe cases (initial GCS scores between 3 and 8), and 100 were considered moderate cases (initial GCS scores between 9 and 12). Of the 287 patients treated with LMWH, 21 (7.3\%) (11 severe TBI and 10 moderate TBI) developed VTEs, and importantly, none of these patients, including patients diagnosed with PEs, died from these events. Only 9 patients $(3.1 \%)$ developed proximal VTEs. Table 1 shows a breakdown of the characteristics of all patients included in the study, as well as these same characteristics for the patients who developed VTEs and those who did not. None of these characteristics were statistically significantly different between the two groups. A clinically im- portant trend was seen for a greater percentage of males in the VTE group compared to the non-VTE group $(90.5 \%$ versus $73.3 \%)$, but this did not achieve statistical significance $\left(\chi^{2}{ }_{1 \mathrm{df}}=3.025, p=0.082\right)$.

One-hundred eighty-six (64.8\%) patients had a brain CT scan performed at least once within 3 weeks after starting LMWH, with a mean of $5.1( \pm 4.5)$ days after, and a median of 3 days. Only one $(0.4 \%)$ patient had an expansion of a preexisting ICH, but this directly led to this patient's death. The single patient who suffered this unfortunate event was an 80year-old woman admitted with a small $(<1 \mathrm{~cm})$, non-surgical subdural hematoma (SDH). She was started on enoxaparin between 48 and $72 \mathrm{~h}$ post-trauma. On post-trauma day 15, almost 2 weeks after starting LMWH, she deteriorated and a $\mathrm{CT}$ scan revealed an acute expansion of her SDH and a significant midline shift. Based on her clinical examination and overall functional status she was not considered a surgical candidate, and she died as a result of this event.

Table 2 compares the baseline characteristics of the group treated with enoxaparin with the group treated with dalteparin. The average initial GCS score was lower in the dalteparin group (6.9 versus $\left.8.0, \mathrm{t}_{285 \mathrm{df}}=3.021, p=0.003\right)$. In addition, the mean ISS was higher (31.06 versus 34.99, $\mathrm{t}_{285 \mathrm{df}}=3.176, p=0.002$ ), and the number of patients requiring an IVC filter was greater $\left(10.9 \%\right.$ versus $23.3 \%, \chi^{2}{ }_{1 \mathrm{df}}=7.381$, $p=0.007)$ in the dalteparin group than in the enoxaparin group. Taken together, these results suggest that the patients in the dalteparin group were more severely injured than those in the enoxaparin group. By breaking down the analysis further into severity groups according to GCS score, we found 
Table 2. Patient Characteristics According to Low-Molecular-Weight Heparin Agent Used

\begin{tabular}{|c|c|c|c|}
\hline & Dalteparin & Enoxaparin & Test and $\mathrm{p}$ value \\
\hline Number of treated patients & $159(55.4 \%)$ & $128(44.6 \%)$ & \\
\hline \multicolumn{4}{|l|}{ Gender } \\
\hline Male & $115(72.3 \%)$ & $99(77.3 \%)$ & $\chi_{1 \mathrm{df}}^{2}=0.941$ \\
\hline Female & $44(27.7 \%)$ & $29(22.7 \%)$ & $\begin{aligned} p & =0.332 \\
t_{285} & =0.626\end{aligned}$ \\
\hline Average age (years) & $45.9 \pm 20.4$ & $47.4 \pm 20.7$ & $\begin{aligned} p & =0.532 \\
t_{285} & =3.021\end{aligned}$ \\
\hline Average initial GCS score & $6.9 \pm 3.2$ & $8.0 \pm 2.8$ & $\begin{aligned} p & =0.003 \\
\mathrm{t}_{285} & =3.176\end{aligned}$ \\
\hline $\begin{array}{l}\text { Average Injury Severity Score } \\
\text { Mechanism of injury }\end{array}$ & \multicolumn{2}{|c|}{ Mechanism of injury } & $p=0.002$ \\
\hline Motor vehicle crash & $63(39.6 \%)$ & $40(31.3 \%)$ & $\chi_{4 \mathrm{df}}^{2}=5.290$ \\
\hline Fall & $46(28.9 \%)$ & $52(40.6 \%)$ & $p=0.259$ \\
\hline Motor vehicle hit & $31(19.5 \%)$ & $20(15.6 \%)$ & \\
\hline Assault & $15(9.4 \%)$ & $14(10.9 \%)$ & \\
\hline Other & $4(2.5 \%)$ & $2(1.6 \%)$ & \\
\hline Polytrauma & $114(71.7 \%)$ & $84(65.6 \%)$ & $\chi_{1 \mathrm{df}}^{2}=1.223$ \\
\hline Isolated head injury & $45(28.3 \%)$ & $44(33.4 \%)$ & $p=0.269$ \\
\hline Any surgery & $112(70.4 \%)$ & $80(62.5 \%)$ & $\begin{aligned} \chi_{1 \mathrm{df}}^{2} & =2.019 \\
p & =0.155\end{aligned}$ \\
\hline Prophylactic IVC filter & $37(23.3 \%)$ & $14(10.9 \%)$ & $\begin{aligned} \chi_{1 \mathrm{df}}^{2} & =7.381 \\
p & =0.007\end{aligned}$ \\
\hline Intracranial bleed while treated & $0(0.0 \%)$ & $1(0.08 \%)$ & \\
\hline
\end{tabular}

GCS, Glasgow Coma Scale; IVC, inferior vena cava.

that only the severe group (GCS score 3-8) had this statistically significant higher percentage of prophylactic IVC filter placements in the dalteparin group than in the enoxaparin group $\left(28.7 \%\right.$ versus $\left.11.7 \%, \chi_{1 \mathrm{df}}^{2}=6.300, p=0.012\right)$.

We compared patients treated with enoxaparin to those treated with dalteparin in terms of development of VTEs and the locations of those VTEs (e.g., above-the-knee DVTs and PEs). In the dalteparin group, $7.5 \%$ experienced VTEs, compared to $7.0 \%$ in the enoxaparin group (Fig. 2), which was not statistically significant $\left(\chi^{2}{ }_{1 \mathrm{df}}=0.028, p=0.868\right)$. It is clinically relevant to note, however, that one patient treated with dal- teparin developed an internal jugular vein DVT, and another three patients treated with dalteparin developed PEs, while no patients treated with enoxaparin developed internal jugular vein DVTs or PEs. In total, 6 (3.8\%) patients treated with dalteparin developed a proximal VTE, while $3(2.3 \%)$ patients treated with enoxaparin developed a proximal VTE (Fig. 3). This also was not statistically significant $\left(\chi^{2} 4 \mathrm{df}=4.200\right.$, $p=0.380)$.

In order to control for the differences in baseline characteristics between the two groups, we used the prophylactic IVC filter as a control indicator, and the ISS score as a

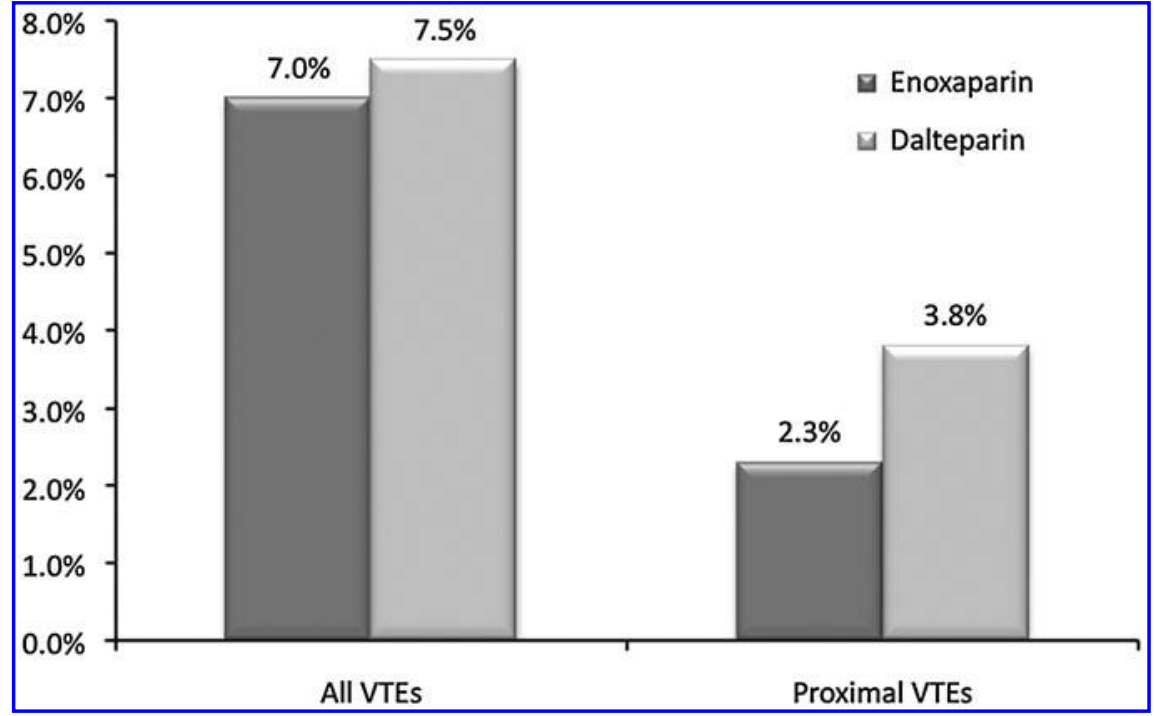

FIG. 2. Percentage of patients on enoxaparin or dalteparin prophylaxis who developed any venous thromboembolic event (VTE, left bars) and proximal VTEs (right bars). 


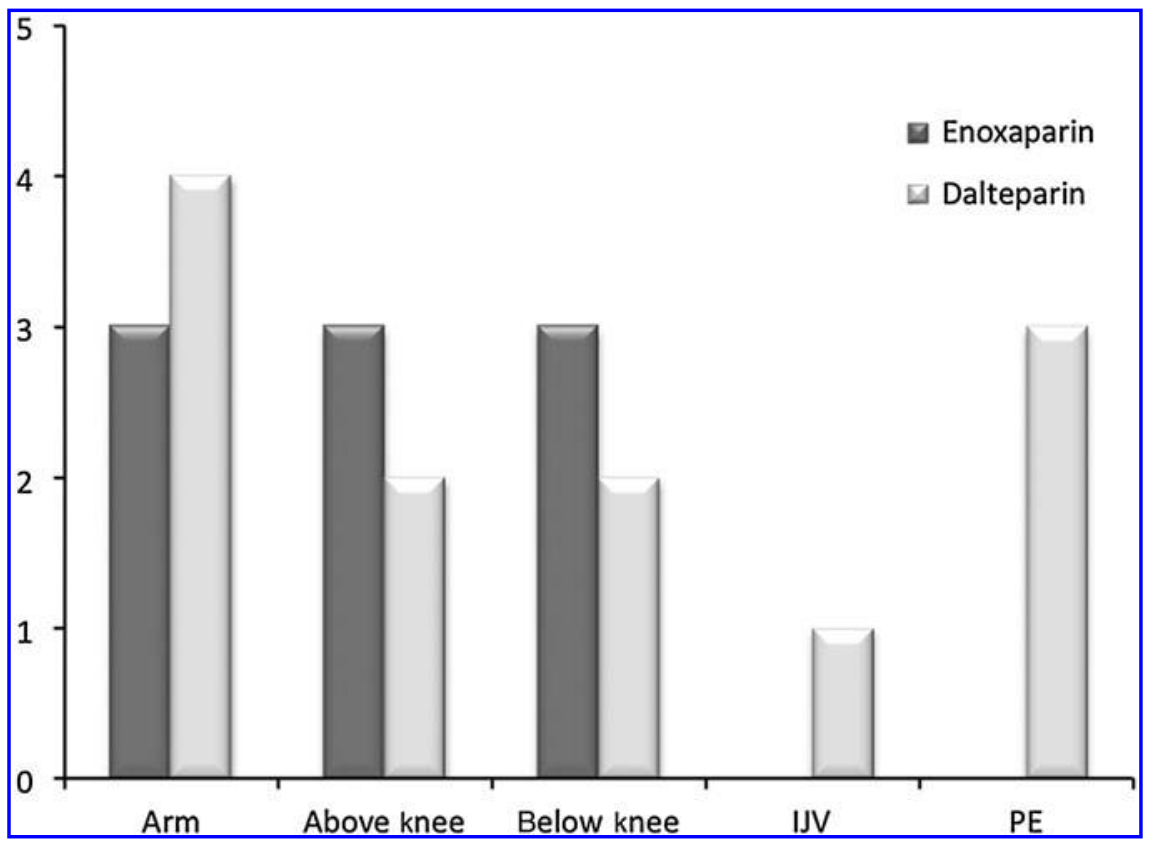

FIG. 3. Number of patients on enoxaparin or dalteparin who developed venous thromboembolic events (VTEs), according to anatomical site (IJV, internal jugular vein; PE, pulmonary embolism).

covariable, as well as their interaction term, to determine if there were medication-associated differences in VTE development, once these confounding variables were controlled for. As indicated by the chi-square Wald statistic, the two groups did not show significant differences in the development of VTE when controlling for ISS and IVC filters (Wald $\left.\chi_{1 \mathrm{df}}^{2}=0.052, p=0.819\right)$. The same results were observed when we used only the distal VTE as a dependent variable (Wald $\chi_{1 \mathrm{df}}^{2}=0.149, p=0.699$ ).

Finally, we examined the timing of VTE diagnosis in our LMWH-treated moderate-to-severe TBI population. Figure 4 shows the distribution in time. The difference in timing of the VTE diagnosis between the dalteparin and enoxaparin groups (mean \pm standard deviation, $20.1 \pm 24.8$ days and $15.6 \pm 7.9$ days, respectively) was not statistically significant $\left(\mathrm{z}_{\text {Mann-Whitney }}=0.454, p=0.464\right)$.

\section{Discussion}

The Brain Trauma Foundation Guidelines (2007) recommend LMWH (or UFH) VTE prophylaxis for TBI patients, but state that there is an increased risk of expansion of $\mathrm{ICH}$, and acknowledges that there is insufficient evidence to support any one particular agent, dose, or timing of administration. Here we have presented a 5-year retrospective case review of LMWH VTE prophylaxis in moderate-to-severe TBI patients from our level 1 trauma center. A small number of previous studies have investigated LMWH use in TBI patients (Kim and Brophy 2009; Kleindienst et al., 2003; Norwood et al., 2002, 2008). However, we believe our study is novel for two reasons. First, it specifically addresses moderate-to-severe TBI patients, while the previous studies either included all TBI patients, or did not comment on injury severity. In particular, the studies of Norwood and colleagues $(2002,2008)$ clearly included patients with mild TBI, as their patient characteristics tables state that patients had GCS scores on admission ranging from 3-15. The study by Kleindienst and associates (2003) appears to have included all patients admitted "because of head injury," and no part of the study, including the exclusion criteria, commented on severity. Finally, in the study by Kim and Brophy (2009), the authors stated that "details of TBI severity were not recorded." We believe the question at hand applies most appropriately to the moderateto-severe subgroup, due to their more prolonged period of immobilization, unlike mild TBI patients (i.e., those with GCS scores 13-15), who are mobilized earlier. The second novel aspect of our study is that the parallel use of enoxaparin and dalteparin as VTE prophylaxis in our hospital over the last

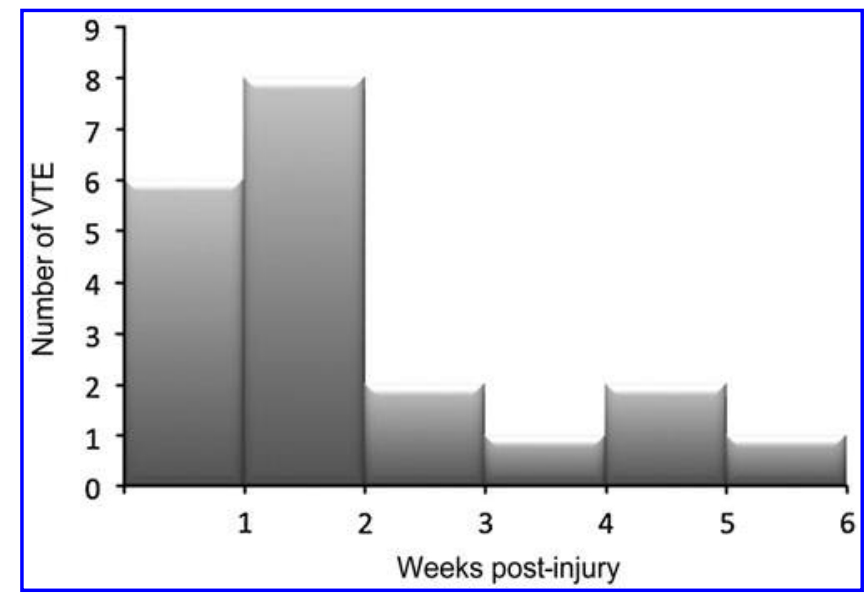

FIG. 4. Number of patients on low-molecular-weight heparin prophylaxis who developed venous thromboembolic events (VTEs, $y$ axis), according to the time elapsed since the traumatic event (in weeks, $x$ axis). One patient had a VTE diagnosed in the 13th week. 
several years afforded us the opportunity to directly compare these medications in TBI patients.

The most important finding of this study is that LMWH (in addition to mechanical prophylaxis), when started at 48-72 hours post-trauma in non-ambulatory patients (with no confounding coagulopathy, and ICH stability on consecutive CT scans), was associated with a low incidence of VTEs (7.3\% VTE rate and $3.1 \%$ proximal VTE rate), and was safe, with only one patient $(0.4 \%)$ having a symptomatic expansion of a pre-existing ICH.

The rate of VTEs in TBI patients has been reported to be as high as $20-25 \%$ when medical prophylaxis is not used or is significantly delayed (i.e., beyond 1 week; Denson et al., 2007; Kaufman et al., 1983). In previous studies addressing the use of prophylactic LMWH in TBI, the rate of VTE was between 0 and $4 \%$ when medications were started at $24 \mathrm{~h}$ post-trauma (Kleindienst et al., 2003; Norwood et al., 2002, 2008). One explanation for the lower rate of VTEs in these previous reports compared to our study may be that they did not stratify patients by TBI severity, while we included only patients with moderate-to-severe TBI (GCS scores 3-12). Therefore, some of these previous studies included patients with mild injuries who were more likely to ambulate earlier, and thus would have been less susceptible to VTEs.

However, a more salient point with regard to the lower rate of VTEs seen in these previous studies compared to ours is the difference in timing of commencement of VTE prophylaxis between our study and these previous reports (Cothren et al., 2007; Kleindienst et al., 2003; Norwood et al., 2002, 2008). In all of these prior studies LMWH was started at $24 \mathrm{~h}$ posttrauma. Recently, Reiff and associates (2009) looked specifically at this question, and reported an increased risk of DVTs in TBI patients with increasing time to initiation of VTE prophylaxis, with a $3.6 \%$ risk when started within the first $24 \mathrm{~h}$, which increased to $4.5 \%$ when started within $24-48 \mathrm{~h}$, and a $15.4 \%$ risk if started beyond $48 \mathrm{~h}$ (Reiff et al., 2009). Thus the lower rate of VTEs seen in these previous studies is possibly due to starting the medications earlier than we did in our population. Indeed, when we examined the timing of VTE diagnosis in our population, the great majority of patients were diagnosed in the first 2 weeks post-trauma, suggesting that TBI patients are susceptible to VTEs very early in the course of injury, and should be treated early. This, however, must be balanced against the potential risk of causing or worsening ICH. Indeed, earlier LMWH use (i.e., $<48 \mathrm{~h}$ ) in TBI patients appears to be associated with an increased risk of progression of ICH. The rate of worsening ICH in one of these studies, in which LMWH was started at $24 \mathrm{~h}$ (Norwood et al., 2002), was $4 \%$ for non-operated patients, and as high as $9 \%$ for patients who had been treated with craniotomy after starting enoxaparin. This prompted a protocol change during this previous study, to withhold enoxaparin for $24 \mathrm{~h}$ after the operative procedure. We believe the time point of $48-72 \mathrm{~h}$ to begin LMWH that we used is a reasonable compromise, that allows for a low rate of VTEs, in addition to a very low rate of expansion of ICH. VTE prophylaxis with LMWH is also often difficult to start within the first $24-48 \mathrm{~h}$ following the admission of a trauma patient, since this patient population often requires multiple procedures in the ICU and/or the operating room during this time period.

The second major focus of this review was to compare enoxaparin and dalteparin VTE prophylaxis in moderate-to- severe TBI patients. Ours is the first study to compare these two medications in TBI patients, but two previous studies of spinal cord injury and major orthopedic injury compared enoxaparin to dalteparin (Chiou-Tan et al., 2003; Slavik et al., 2007). Neither of these studies found a statistically significant difference in the percentage of VTEs between the two groups. Slavik and colleagues (2007) reviewed 135 trauma cases of major pelvic, femoral head, complex lower extremity fractures, and/or spinal cord injury, and reported a trend of a $1.6 \%$ rate of VTEs with enoxaparin, and a $9.7 \%$ rate with dalteparin, but the difference was not statistically significant. On the contrary, Chiou-Tan and associates (2003) performed a prospective, randomized study of 100 acute spinal cord injury patients and reported an even smaller, reversed trend, of a $6.0 \%$ rate and a $4.0 \%$ rate of VTEs with enoxaparin and dalteparin, respectively, which again was not statistically significant. Likewise, in our present study we found no difference in the development of VTEs between the enoxaparin- and dalteparin-treated groups. However, in our study these two groups differed in their baseline characteristics, with the dalteparin group having lower GCS scores, higher ISSs, and a greater number of patients with IVC filters implanted, which suggested that the dalteparin-treated patients happened to be more severely injured. When controlling for these differences in baseline characteristics, we found no significant difference in the numbers of VTEs between the dalteparin and enoxaprin groups.

The limitations of this chart review include all those of any retrospective study (Hess, 2004). Specifically for this study, it was not possible to ascertain from the charts exactly how long LMWH was held for various surgeries and other small ICU procedures. For the most part, this appeared to range from 6-12 h, but we could not be certain in all cases, and thus this was not accounted for in our analysis. Also, CT scans of the brain to detect hemorrhage expansion, or other diagnostic exams to detect VTE, were not performed systematically for all patients, but rather only as clinically indicated. Another limitation is that bleeding at other sites, another potential complication of the use of LMWH in trauma patients, was not felt to be reliably documented, and therefore was not accounted for in this study. Despite these drawbacks, we believe this study, with its relatively large number of moderate-to-severe TBI patients, contributes important data regarding the use of LMWH in these patients, and provides the first direct comparison between enoxaparin and dalteparin in this population.

In conclusion, the results of this study suggest that $\mathrm{LMWH}$ started at $48-72 \mathrm{~h}$ in non-ambulatory patients, with no confounding coagulopathy and at least two CT scans revealing ICH stability, is a safe and effective means of decreasing the rates of VTEs in moderate-to-severe TBI patients. They also suggest that there was no difference in VTE rates between dalteparin- and enoxaparin-treated patients.

\section{Acknowledgments}

We would like to thank Mrs. Johanne Prud'homme from the Trauma Registry of the Montreal General Hospital for quickly providing the necessary data. We also want to thank Mrs. Mitra Feyz, Director of the Traumatic Brain Injury Program, for providing the data from the Traumatic Brain Injury Data Bank, and for her continuous support. 


\section{Author Disclosure Statement}

No competing financial interests exist.

\section{References}

Brain Trauma Foundation; American Association of Neurological Surgeons; Congress of Neurological Surgeons; Joint Section on Neurotrauma and Critical Care, AANS/CNS, Bratton S.L., Chestnut, R.M., Ghajar, J., McConnell Hammond, F.F., Harris, O.A., Hartl, R., Manley, G.T., Nemecek, A., Newell, D.W., Rosenthal, G., Schouten, J., Shutter, L., Timmons, S.D., Ullman, J.S., Videtta, W., Wilberger, J.E., and Wright, D.W. (2007). Guidelines for the management of severe traumatic brain injury. V. Deep vein thrombosis prophylaxis. J. Neurotrauma 24, S32-S366.

Chiou-Tan, F.Y., Garza, H., Chan, K.T., Parsons, K.C., Donovan, W.H., Roberston, C.S., Holmes Graves, D.E., and Rintala, D.H. (2003). Comparison of dalteparin and enoxaparin for deep venous thrombosis prophylaxis in patients with spinal cord injury. Am. J. Phys. Med. Rehabil. 82, 678-687.

Cothren, C.C., Smith, W.R., Moore, E.E., and Morgan, S.J. (2007). Utility of once-daily dose of low-molecular-weight heparin to prevent venous thromboembolism in multisystem trauma patients. World J. Surg. 31, 98-104.

Denson, K., Morgan, D., Cunningham, R., Nigliazzo, A., Brackett, D., Lane, M., Smith, B., and Albrecht, R. (2007). Incidence of venous thromboembolism in patients with traumatic brain injury. Am. J. Surg. 193, 380-383.

Geerts, W.H., Jay, R.M., Code, K.I., Chen, E., Szalai, J.P., Saibil, E.A., and Hamilton, P.A. (1996). A comparison of low-does heparin with low-molecular-weight heparin as prophylaxis against venous thromboembolism after major trauma. $\underline{\mathrm{N}}$. Engl. J. Med. 335, 701-707.

Geerts, W.H., Pineo, G.F., Heit, J.A., Bergqvist, D., Lassen, M.R., Colwell, C.W., and Ray, J.G. (2004). Prevention of venous thromboembolism: the Seventh ACCP Conference on Antithrombotic and Thrombolytic Therapy. Chest 126, 338S-400S.

Gerlach, R., Scheuer, T., Beck, J., Woszczyk, A., Seifert, V., and Raabe, A. (2003). Risk of postoperative hemorrhage after intracranial surgery after early nadroparin administration: results of a prospective study. Neurosurgery 53, 1028-1034.

Hess, D.R. (2004). Retrospective studies and chart reviews. Respir. Care 49, 1171-1174.

Kaufman, H.H., Satterwhite, T., McConnell, B.J., Costin, B., Borit, A., Gould, L., Pruessner, J., Bernstein, D., and Gildenberg, P.L. (1983). Deep vein thrombosis and pulmonary embolism in head injured patients. Angiology 34, 627-638.

Kim, K.S., and Brophy, G.M. (2009). Symptomatic venous thromboembolism: incidence and risk factors in patients with spontaneous or traumatic intracranial hemorrhage. Neurocrit. Care 11, 28-33.
Kleindienst, A., Harvey, H.B., Mater, E., Bronst, J., Flack, J., Herenz, K., Haupt, W.F., and Schön, R. (2003). Early antithrombotic prophylaxis with low molecular weight heparin in neurosurgery. Acta Neurochir. (Wien.) 145, 1085-1090.

Nathens, A.B., McMurray, M.K., Cuschieri, J., Durr, E.A., Moore, E.E., Bankey, P.E., Freeman, B., Harbrecht, B.G., Johnson, J.L., Minei, J.P., McKinley, B.A., Moore, F.A., Shapiro, M.B., West, M.A., Tompkins, R.G., and Maier, R.V. (2007). The practice of venous thromboembolism prophylaxis in the major trauma patient. J. Trauma 62, 557-562.

Norwood, S.H., Berne, J.D., Rowe, S.A., Villarreal, D.H., and Ledlie, J.T. (2008). Early venous thromboembolism prophylaxis with enoxaparin in patients with blunt traumatic brain injury. J. Trauma 65, 1021-1026.

Norwood, S.H., McAuley, C.E., Berne, J.D., Vallina, V.L., Kerns, D.B., Grahm, T.W., Short, K., and McLarty, J.W. (2002). Prospective evaluation of the safety of enoxaparin prophylaxis for venous thromboembolism in patients with intracranial hemorrhagic injuries. Arch. Surg. 137, 696-701.

Reiff, D.A., Haricharan, R.N., Bullington, N.M., Griffin, R.L., McGwin, G. Jr., and Rue, L.W. 3rd. (2009). Traumatic brain injury is associated with the development of deep vein thrombosis independent of pharmacological prophylaxis. I. Trauma 66, 1436-1440.

Rogers, F.B., Cipolle, M.D., Velmahos, G., Rozycki, G., and Luchette, F.A. (2002). Practice management guidelines for the prevention of venous thromboembolism in trauma patients: the EAST practice management guidelines work group. I. Trauma 53, 142-164.

Slavik, R.S., Chan, E., Gorman, S.K., de Lemos, J., Chittock, D., Simons, R.K., Wing, P.C., and Ho, S.G. (2007). Dalteparin versus enoxaparin for venous thromboembolism prophylaxis in acute spinal cord injury and major orthopedic trauma patients: DETECT trial. J. Trauma 62, 1075-1081.

Smith, R.M., Airey, M., and Franks, A.J. (1994). Death after major trauma: can we affect it? The changing cause of death in each phase after injury [abstract]. Injury 25, SB23-SB24.

Teasdale, G., and Jennett, B. (1974). Assessment of coma and impaired consciousness: a practical scale. Lancet 13, 81-84.

Address correspondence to: Judith Marcoux, M.D., M.Sc., FRCSC Department of Neurosurgery Montreal General Hospital McGill University Health Centre 1650 Cedar Avenue, Room L7-524 Montreal, Quebec, Canada, H3G 1 A4

E-mail: judith.marcoux@mcgill.ca 
This article has been cited by:

1. George A. Alexiou , Dimitrios Pahatouridis, Spyridon Voulgaris . Coagulation Disorders in Traumatic Brain InjuryCoagulation Disorders in Traumatic Brain Injury. Journal of Neurotrauma, ahead of print. [Citation] [Full Text] [PDF] [PDF Plus] 